\title{
A NEW CHARACTERIZATION OF PLANAR GRAPHS
}

\author{
BY C. H. C. LITTLE AND D. A. HOLTON
}

Communicated by Walter Gautschi, April 4, 1976

We consider graphs on a finite set of vertices. In addition the graphs are undirected, although, for the purposes of the characterization, we will need to give each edge a direction. We use the notation $E G$ to denote the set of edges of the graph $G$, and $V G$ for the corresponding vertex set. A graph is defined to be planar if and only if it can be embedded in the plane so that any two edges intersect at a common end-vertex or not at all.

Our characterization of planar graphs is given by the following theorem. For other characterizations, see [1].

THEOREM 1. A graph is nonplanar if and only if it contains a maximal, strict, compact, odd ring.

We now explain the terms introduced in the characterization.

Let $S$ be a collection of $n$ circuits in a graph $G$ and suppose that the edges of $G$ may be directed so that each circuit of $S$ is a directed circuit. We say that, for $n \geqslant 3, S$ is a ring if (i) the circuits of $S$ can be labelled $C_{0}, C_{1}, \ldots, C_{n-1}$ so that $E C_{i} \cap E C_{j} \neq \varnothing$ if and only if $i=j, i \equiv j+1 \bmod n$ or $i \equiv j-1 \bmod n$, and (ii) no edge of $G$ belongs to more than two circuits of $S$.

(We note that (i) implies (ii) except when $n=3$.)

The ring $S$ is said to be strict if $\left|V C_{i} \cap V C_{j}\right| \leqslant 1$ whenever $E C_{i} \cap E C_{j}=\varnothing$; it is said to be maximal if there does not exist a ring $\left\{C_{0}^{\prime}, C_{1}^{\prime}, \ldots, C_{m-1}^{\prime}\right\}$ in $G$ such that $\bigcup_{k=0}^{m-1} E C_{k}^{\prime} \subseteq \bigcup_{l=0}^{n-1} E C_{l}$ and $m>n$. If $n$ is odd, then $S$ is said to be odd. Finally, $S$ is compact if there is no ring $\left\{C_{0}^{\prime \prime}, C_{1}^{\prime \prime}, \ldots, C_{n-1}^{\prime \prime}\right\}$ such that $\bigcup_{k=0}^{n} E C_{k}^{\prime \prime} \subset \bigcup_{l=0}^{n} E C_{l}$.

The characterization is proved by purely combinatorial means which are motivated by topological considerations. We set out the main steps of the proof in the sequence of lemmas below.

LEMMA 1. Every nonplanar graph contains a maximal, strict, compact, odd ring.

The proof of this lemma follows from [2], where a maximal, odd ring is constructed in every nonplanar graph.

AMS (MOS) subject classifications (1970). Primary $05 \mathrm{C} 10$. 
LEMMA 2. If $G$ contains a maximal ring with just three circuits then $G$ is nonplanar.

As a result of this lemma we are free to concentrate on odd rings with five or more circuits. We then show that no two consecutive circuits in our ring may have more than one nontrivial path in common.

LEMMA 3. If $G$ contains a maximal, strict, compact, odd ring with more than three circuits in which some pair of circuits $C_{i}, C_{i+1}$ have more than one nontrivial path in common, then $G$ is nonplanar.

The final lemma concludes the proof of the characterization.

LEMMA 4. If $G$ contains a maximal, strict, compact, odd ring with more than three circuits in which any pair of circuits $C_{i}, C_{i+1}$ have precisely one nonempty path in common, then $G$ is nonplanar.

\section{REFERENCES}

1. F. Harary, Graph theory, Addison-Wesley, Reading, Mass., 1969, pp. 108-116. MR 41 \#1566.

2. C. H. C. Little, A conjecture about circuits in planar graphs, Lecture Notes in Math., vol. 452, Springer-Verlag, Berlin and New York, 1975, pp. 171-175.

DEPARTMENT OF MATHEMATICS AND COMPUTER SCIENCE, ROYAL MELBOURNE INSTITUTE OF TECHNOLOGY, MELBOURNE, AUSTRALIA

DEPARTMENT OF MATHEMATICS, UNIVERSITY OF MELBOURNE, PARKVILLE, VICTORIA, AUSTRALIA 